\title{
Synergic Antitumor and Antitoxicity Effects of the Traditional Uyghur Medicine Abnormal Savda Munziq when Combined with 5-Fluorouracil
}

\author{
Ainiwaer Aikemu \\ (First author) \\ Central Laboratory \\ Xinjiang Medical University \\ Urumqi, Xinjiang, China \\ e-mail: ainiwa@sina.com \\ YingYing Liu \\ Department of Oncology \\ Traditional Chinese Medicine Hospital Affiliated \\ to Xinjiang Medical University \\ Urumqi, Xinjiang, China \\ e-mail:987356231@qq.com

\section{Zhen Gao} \\ National Clinical Research Base of Traditional \\ Chinese Medicine of Xinjiang \\ Traditional Chinese Medicine Hospital Affiliated \\ to Xinjiang Medical University \\ Urumqi, Xinjiang, China \\ e-mail: gaozhening@gmail.com
}

Abstract-Objective: A number of traditional Uyghur medicines have been recognized as potent antitumor agents and have been developed and analyzed in vivo. In the present study, the toxic, therapeutic and cytotoxic properties of the Uyghur medicine Abnormal Savda Munziq (ASMq) were investigated. In the presence of certain agents, ASMq results in the fragmentation of plasmid DNA, indicating that it may be a potent antitumor agent in vivo, as it appears to bind to DNA molecules. Thus, the present study investigated the effects of ASMq in combination with 5-fluorouracil (5-FU) in vivo, using an S180 mouse model of cancer. ASMq was observed to enhance the antitumor effect of 5-FU and also attenuated the cytotoxicity of the oxidative stress reactions. Methods: The mice were divided randomly into six groups. Influence of treatment on the activity of mice were observed. The serum levels of SOD, MDA, GSH-Px and pathological change were determined. Results: Compared with the model group, the data turned normal after gavaged 5-FU and ASMq. Conclusion: In summary, a combination of ASMq and 5-FU resulted in a synergistic antitumor effect and attenuated toxicity, suggesting that ASMq may be clinically applicable for the treatment of certain types of cancer.

Keywords-Traditional Uyghur Medicine; Abnormal Savda Munziq; 5-Fluorouracil; Antitumor Effects; Antitoxicity Effects

\author{
Lianlian Shan \\ Department of Drug Analysis \\ Xinjiang Medical University \\ Urumqi, Xinjiang, China \\ e-mail: happyshanlian@sina.com \\ Aerziguli Tuerxun \\ Clinical Medical Research Institute \\ The First Affiliated Hospital of Xinjiang \\ Medical University \\ Urumqi, Xinjiang, China \\ e-mail: 396737815@qq.com \\ Halmurat Upur* \\ (Corresponding author) \\ Traditional Uighur Medicine and Pharmacology \\ Xinjiang Medical University \\ Urumqi, Xinjiang, China \\ e-mail: halmurat@263.net
}

\section{INTRODUCTION}

Abnormal Savda Munziq (ASMq) is a traditional Uyghur medicine (TUM) that is widely distributed in the Xinjiang province of China. ASMq is comprised of ten medicinal herbs, including Cordia dichotoma, Euphorbia humifusa, Anchusa italica, Adiantum flabellulatum and Borago officinalis. ASMq has been used as a TUM for various types of cancer, diabetes mellitus, cardiovascular diseases and chronic asthma [1]. ASMq is regularly used by physicians of Uyghur ethnic background for the treatment of cancer, and by numerous individuals in the south of Xinjiang as a preventative self-medication for the prevention of certain types of cancer.

Previous studies have investigated ASMq using molecular biological and pharmacological methods: One study identified that ASMq may 'mop up' free radicals and superoxide anions, preventing oxidative damage to DNA [2]. In addition, other studies have suggested that ASMq may exert free radical scavenging effects, reduce the concentration of oxidative stress biological markers in humans, protect mitochondria and DNA against $\mathrm{OH}$-induced oxidative damage in cell-free systems and inhibit the proliferation of cancer cells in vitro [3-6].5-Fluorouracil (5-FU) is a structural analog of the 
pyrimidines uracil and thymidine. 5-FU contains a fluorine atom substituted in the carbon-5 position and modulates the enzyme thymidylate synthetase, which in turn blocks the synthesis of DNA. 5-FU has been employed successfully in the treatment of gastrointestinal malignancies, carcinoma of the aero-digestive tract, breast cancer and bladder cancer [7,8]. In the present study, the effects of ASMq combined with 5-FU were investigated in vivo using an S180 cancer mouse model. It was hypothesized that ASMq may enhance the antitumor effects of 5-FU, and attenuate the associated levels of oxidative stress.

\section{MATERIALS AND METHODS}

\section{A. Ethical approval of the study protocol}

The present study protocol was approved by the Ethics Committee of Xinjiang Medical University (Urumqi, China).

\section{B. Reagents}

ASMq was obtained as a granular formulation from Xinjiang Qikang Habo Uyghur Medicine Co., Ltd. (Urumqi, China; Batch Number: 06060). 5-FU was obtained from Tianjin Jinyao Amino Acid Co., Ltd. (Tianjin, China; Batch Number: 0912302). The physiological saline was obtained from Sinopharm Xinjiang Pharmaceutical Co., Ltd. (Batch number: 11030831). The Superoxide dismutase (SOD) kit was purchased from the Nanjing Jiancheng Bioengineering Institute (Nanjing, China; Batch Number: 20100420). The malondialdehyde (MDA) kit was purchased from the Nanjing Jiancheng Bioengineering Institute (Nanjing, China; Batch Number: 2100420) and the glutathione peroxidase (GSH-Px) kit was purchased from the Nanjing Jiancheng Bioengineering Institute (Nanjing, China; Batch Number: 20100420).

\section{Animals}

In total, 60 healthy Kunming mice (weight, $20 \pm 2 \mathrm{~g}$; age, 4-6 weeks; gender, 30 males and 30 females) were obtained from Xinjiang Medical University Experimental Animal Center (license no. 2003-001; Urumqi, China).

\section{Preparation of the $S 180$ mouse model}

Kunming mice were bred under standard laboratory conditions at room temperature, with a $12 \mathrm{~h}$ light dark cycle and free access to standard rodent chow and water. The mice were divided randomly into six groups: control group, model group, 5-FU group, high-dose ASMq+5-FU group (ASMq.H), medium-dose ASMq+5-FU group (ASMq.M), low-dose ASMq+5-FU group (ASMq.L), 10 mice per group, each group half male and half female. A total of 60 mice in the control group except for the remaining five groups $(50$ mice $)$ were inoculated with s180 cells.

\section{E. Method}

S180 cells were supplied by Laboratory Animal Centre of Xinjiang military hospital. Cream colored ascites were extracted from the Kunming mouse which was Inoculated seven days old mice under aseptic conditions. Using high pressure antiseptic physiological saline Diluted to the ascites $1 \times 10^{7}$ cells $/ \mathrm{ml}$. With the exception of the control group, $0.2 \mathrm{ml}$ ascites $\left(1 \times 10^{7}\right.$ cells $/ \mathrm{ml}$ ) were transplanted into the right axilla of all the mouse, the entire operation is completed under sterile conditions within 30 minutes. After $24 \mathrm{~h}$, the intervention was continued according to the body weight of the mouse.

The control and model groups were daily injected with $(0.2 \mathrm{ml} / 10 \mathrm{~g}$ body weight $)$ physiological saline (NS) via the intraperitoneal (i.p.) route and $\mathrm{NS}(0.2 \mathrm{ml} / 10 \mathrm{~g}$ body weight) via the intragastric (i.g.) route.The positive control (5-FU) group received 5-FU (i.p, $25 \mathrm{mg} / \mathrm{kg}$ ) on day $1,3,5,7$, and 9 (a total of five doses). The ASMq.H group received 5-FU (i.p , on day 1, 3, 5, 7and 9, 25 $\mathrm{mg} / \mathrm{kg}$ ) + high-dose ASMq (i.g, $8 \mathrm{~g} / \mathrm{kg}$ daily); the ASMq.M group received 5-FU (i.p, on day 1, 3, 5, 7 and 9, for $25 \mathrm{mg} / \mathrm{kg}$ ) + medium-dose ASMq (i.g, $4 \mathrm{~g} / \mathrm{kg}$ daily); and the ASMq.L group received 5-FU (i.p, on day 1, 3, 5, 7 and 9, $25 \mathrm{mg} / \mathrm{kg}$ ) + low-dose ASMq (i.g, $2 \mathrm{~g} / \mathrm{kg}$ daily). The mice were euthanized ten days after the start of the experiment. The thymus, spleen, liver and tumors were removed and weighed respectively.

\section{F. Determination of serum levels $f$ SOD, MDA and GSH-Px}

Following the establishment of the S180 mouse model and continual dosing with ASMq for ten days, blood samples were collected via the eye socket. The samples were centrifuged at 3,000 rpm for $20 \mathrm{~min}$ at room temperature $\left(25^{\circ} \mathrm{C}\right)$ in order to obtain the sera. The serum levels of SOD, MDA, GSH-Px were determined by xanthine oxidase method, thiobarbituric acid (TBA) method and Ellman method according to use the kit manufacturer's instructions strictly.

\section{G. Statistical analysis}

Data are expressed as the $\bar{x} \pm$ SD. SPSS software, version 17.0 (SPSS, Inc., Chicago, IL, USA) was used for analyses. $\mathrm{P}<0.05$ was considered to indicate a statistically significant difference.

\section{RESULTS}

\section{A. Influence of treatment on the activity of mice}

Following treatment, animals'behavior, autonomic activities, food intake, water intake, fur quality, feces and urine have been observed by the naked eye comparison as normal mice behavior. The model group exhibited reduced activity, enlarged tumors, unclean fur and listlessness, with a tumor growth incubation period (TT) of two days and the fastest tumor growth velocity (TS) of the groups. The ASMq.L group exhibited normal activity, good 
mental state and clean fur, with a TT of three days and increased TS compared with the model group. The ASMq.M group exhibited normal activity, with an improved mental state and fur cleaning behavior compared with the ASMq.L group. Furthermore, the TT was three days and TS was reduced compared with the ASMq.L group. The ASMq.H group also displayed normal activity, with a mental state and fur cleaning behavior that was increased compared with the ASMq.L and ASMq.M groups. The ASMq.H TT was three days and the TS was reduced compared with the ASMq.L and ASMq.M groups. In the 5-FU group, normal activity and a good mental state and clean fur were observed, which were improved compared with the ASMq.L group. The TT was four days and the TS was the lowest of the groups.

\section{B. Influence of treatment on weight of internal organs/body weight}

Subsequent to the processing of tissue samples from the thymus, spleen and liver, the mass ratio of these internal organs was calculated using the following formula: Mass ratio = organ weight $(\mathrm{g}) /$ body weight $(\mathrm{g})$.
Compared with the model, in the ASMq.H, ASMq.M, ASMq.L and the 5-FU groups, tumors were reduced in size, indicating that the normal functions of the tumors were weakened. The thymus weight in the ASMq.H, ASMq.M and ASMq.L groups were increased comparing with the 5-FU group $(\mathrm{P}<0.05)$. Compared with the model and 5-Fu group, in the ASMq.M group the spleen weight were increased $(\mathrm{P}<0.05)$, in the ASMq.L groups the liver weight were increased $(\mathrm{P}<0.05)$. (Table I.)

The tumor inhibition rate values in the 5-FU, ASMq.H, ASMq.M and ASMq.L and groups were 48.02, $52.26,66.41$ and $44.87 \%$, respectively, indicating that tumor function had been inhibited by these treatments. An evident increase was observed in the thymus/body weight ratio of the ASMq.H and ASMq.L groups compared with the model group $(\mathrm{P}<0.05)$. A statistically significant increase was observed in the spleen/body weight ratio of the ASMq.H, ASMq.M and ASMq.L groups compared with the 5-FU group $(\mathrm{P}<0.05)$. Compared with the model group and the 5-FU group, the liver/body weight ratio of the ASMq.H group was decreased, in the ASMq.L group was increased $(\mathrm{P}<0.05)$. (Table II.)

TABLE I . ANTITUMOR ACTIVITY OF ASMq IN AN S180 TUMOR MOUSE MODEL ( $\bar{x} \pm$ SD)

\begin{tabular}{cccccc}
\hline Group & Tumor $(\mathrm{g})$ & Thymus $(\mathrm{g})$ & Spleen $(\mathrm{g})$ & Liver $(\mathrm{g})$ & Weight $(\mathrm{g})$ \\
Control & $0.00 \pm 0.00$ & $0.10 \pm 0.02$ & $0.10 \pm 0.01$ & $1.20 \pm 0.05$ & $30.65 \pm 2.61$ \\
Model & $0.57 \pm 0.03$ & $0.09 \pm 0.03$ & $0.11 \pm 0.01$ & $1.20 \pm 0.05$ & $29.64 \pm 0.84$ \\
5 -Fu & $0.29 \pm 0.02$ & $0.06 \pm 0.01$ & $0.08 \pm 0.01$ & $1.14 \pm 0.04$ & $31.25 \pm 0.65^{* \Delta}$ \\
ASMq.H & $0.26 \pm 0.03^{* \Delta}$ & $0.11 \pm 0.01^{\Delta}$ & $0.11 \pm 0.01^{\Delta}$ & $1.16 \pm 0.10$ & $1.33 \pm 0.36$ \\
ASMq.M & $0.19 \pm 0.02^{* \Delta}$ & $0.09 \pm 0.01^{\Delta}$ & $0.12 \pm 0.02^{* \Delta}$ & $30.39 \pm 1.30^{\Delta}$ \\
ASMq.L & $0.31 \pm 0.01^{* \Delta}$ & $0.10 \pm 0.02^{\Delta}$ & $0.11 \pm 0.02^{\Delta}$ & $1.35 \pm 0.18^{*}$ & $26.55 \pm 0.67^{* \Delta}$ \\
\hline
\end{tabular}

Note: $* \mathrm{P}<0.05$, compares with the model group; ${ }^{\Delta} \mathrm{P}<0.05$, compares with the 5-Fu group

TABLE II . INHIBITORY EFFECT OF ASMq ON THE GROWTH OF TRANSPLANTED TUMORS IN S180 MICE AND ORGAN/WEIGHT RATIO

\begin{tabular}{ccccc}
\hline & Thymus/body & Spleen/body & Liver/body weight & Inhibitory rate \\
Group & weight & weight $(\mathrm{g} / \mathrm{g})$ & $(\mathrm{g} / \mathrm{g})$ & $(\%)$ \\
& $0.0035 \pm 0.0007$ & $0.0034 \pm 0.0005$ & $0.0404 \pm 0.0018$ & 48.02 \\
Control & $0.0029 \pm 0.0010$ & $0.0037 \pm 0.0007$ & $0.0415 \pm 0.0041$ & 52.26 \\
Model & $0.0024 \pm 0.0003$ & $0.0030 \pm 0.0004$ & $0.0452 \pm 0.0017$ & 66.41 \\
ASMq.H & $0.0034 \pm 0.0004^{*}$ & $0.0034 \pm 0.0004^{\Delta}$ & $0.0370 \pm 0.0037^{*} \Delta$ & 44.87 \\
ASMq.M & $0.0029 \pm 0.0004^{\Delta}$ & $0.0039 \pm 0.0006^{\Delta}$ & $0.0434 \pm 0.0112$ & \\
ASMq.L & $0.0039 \pm 0.0005^{*}$ & $0.0040 \pm 0.0006^{*} \Delta$ & $0.0506 \pm 0.0066^{*}$ & \\
\hline
\end{tabular}

Note: $* \mathrm{P}<0.05$, compares with the model group; ${ }^{\Delta} \mathrm{P}<0.05$, compares with the 5 -Fu group 


\section{Effect of ASMq treatment on serum levels of SOD,}

\section{$M D A$ and GSH-PX}

The ASMq.M group exhibited the highest levels of SOD and GSH-Px compared with the model group and hard upon to the control group value $(\mathrm{P}<0.05)$. Furthermore, the 5-FU group exhibited significantly lower levels of SOD and GSH-Px comparing with the model group $(\mathrm{P}<0.05)$. The ASMq.M and ASMq.H groups exhibited significantly increased levels of SOD and GSH-Px compared with the 5-FU group $(\mathrm{P}<0.05)$. (Fig .1)

The ASMq.M group displayed significantly lower MDA levels comparing with the model group $(\mathrm{P}<0.05)$. The ASMq groups exhibited significantly reduced levels of MDA comparing with the 5-FU group $(\mathrm{P}<0.05)$. (Fig .2)

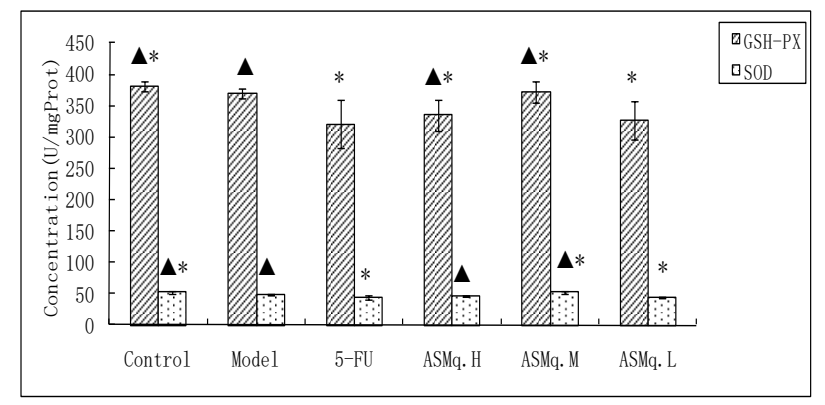

* compared with the model group; ${ }^{\boldsymbol{}}$ compared with the 5-FU group

Figure 1. Effects of ASMq on SOD and GSH-Px

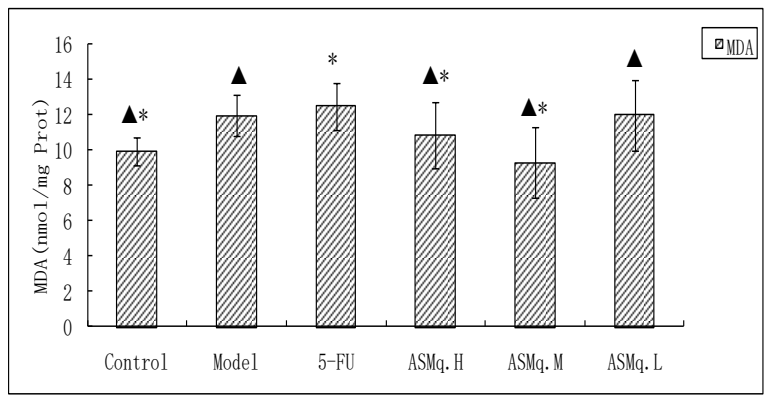

* compared with the model group; ${ }^{\wedge}$ compared with the 5-FU group

Figure 2. Effects of ASMq on MDA

\section{Tumor pathology}

As can be observed in Fig .3, the 5-FU group sections presented clear tumor tissue damage compared with the model group, including large areas of tissue necrosis that was primarily eosinophilic. There were small areas of tissue necrosis in the ASMq.L group, while large areas of tissue necrosis were observable in the ASMq.M and ASMq.H groups. The ASMq.M group exhibited evident ischemic necrosis and phlogocyte infiltration. (Fig .3)

\section{Model group}
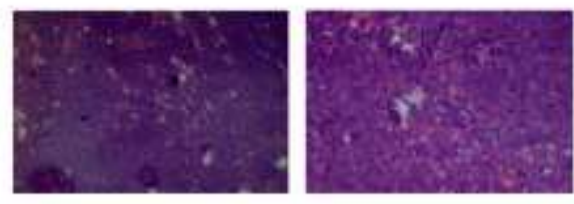

\section{5-FU group}
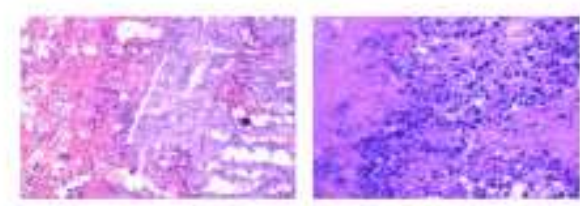

\section{ASMq. \\ L \\ group}
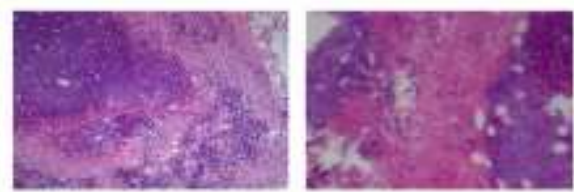

ASMq.
M
group
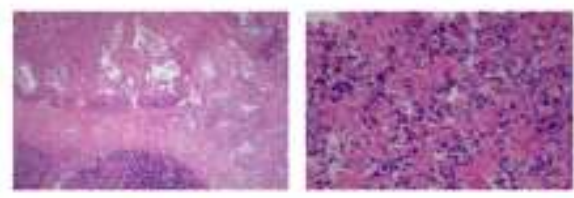

ASMq. H

\section{group}
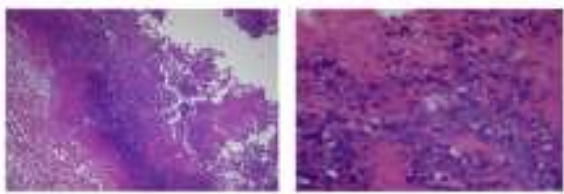

Figure 3. Hematoxylin and eosin staining of tumor sections from the model, 5-FU, ASMq.L, ASMq.M and ASMq.H groups

\section{(Left-column images are presented at magnification, $\mathrm{x} 10$; and right-column images at magnification, $\mathrm{x} 40$.)}

\section{DISCUSSION}

A previous study indicated that ASMq possesses antitumor and immunomodulatory properties [9]. In the present study, ASMq was observed to significantly inhibit the growth of transplanted mouse tumors compared with model mice, and it increased relative spleen weights compared with those receiving 5-FU treatment alone, suggesting that ASMq exerted an antitumor effect and stimulated splenocyte proliferation.

However, in contrast to the notable antitumor and immunomodulatory activities of ASMq in mice, ASMq previously displayed a weak cytotoxic effect on tumor cells in vitro [10]. This contradiction suggests that ASMq may exert an antitumor effect by enhancing immune function rather than by directly attacking cancer cells, in a similar manner to the polysaccharides from mushrooms [11]. Thus, ASMq may possess considerable potential for development as an adjuvant of chemotherapy drugs due to its immunomodulatory activity and low toxicity.

5-FU is one of the most effective chemotherapeutic agents available for treating numerous types of solid tumors [12]. However, the continuous use of 5-FU is not 
always feasible due to cumulative toxicity. Therefore, it is essential to develop new adjuvant therapies that protect patients with cancer from the harmful side effects of chemotherapy, without reducing the efficacy of the treatment. In the present study, the antitumor effect of ASMq in combination with 5-FU was evaluated in a mouse model of S180 cancer. The results indicated that ASMq exerted a significant synergistic antitumor effect in combination with 5-FU. Furthermore, the 5-FU-induced reduction in the relative weight of the thymus and spleen was reversed by the ASMq treatment. These results further demonstrated the association between the antitumor effect of ASMq and its immunomodulatory activity and confirmed its potential for development as an adjuvant to chemotherapy drugs.

Lipid peroxidation is an important mechanism with respect to cell damage. According to free radical theory, the more rapid the metabolic rate of an organism, the higher the production of free radicals and the shorter the lifespan $[13,14]$. Furthermore, the activity of antioxidant enzymes, particularly SOD, has been reported to slow the aging process [15]. It has been reported that SOD slows aging and inhibits oxidative damage [16], and this may be due to irreversible inactivation by its product, hydrogen peroxide. An alternative explanation for this reduction may be the increase in the glycation of SOD [17]; and it was demonstrated in the present study that 5-FU may reduce the activity of SOD and GSH-Px and increase levels of MDA. MDA is the final product of lipid peroxidation of the cell membrane; therefore, MDA levels may reflect the intensity of lipid peroxidation. SOD and GSH-Px are used to evaluate resistance to oxidative damage [18,19]. Studies have suggested that a combined therapy of 5-FU with ASMq may be a clinically applicable adjuvant treatment in combination with cancer chemotherapy [20,21].

In conclusion, ASMq was observed to enhance the antitumor effect of 5-FU and to attenuate the cytotoxicity of associated oxidative stress reactions. A synergistic antitumor effect and attenuated toxicity were obtained using a combined treatment of ASMq with 5-FU. Thus, ASMq may be potentially useful in the clinical treatment of cancer, and future studies should assess the synergistic and attenuated action of ASMq clinically in the treatment of fibrosarcoma(S180).

\section{ACKNOWLEDGMENT}

The present study was supported by grants from the Ministry of Education Changjiang Scholar and Innovative Team Development Program of China (no. IRT0977) and the National Natural Science Foundation of China (no. 30260128 and 81360668).

\section{CONFLICT OF INTEREST}

The authors declare that they have no conflict of interest.

\section{REFERENCES}

[1] Yusup A, Upur H, Baudrimont I, Umar A, Kader T, and et al., "Cytotoxicity of abnormal Savda Munziq aqueous extract in human hepatoma (HepG2) cells," Fundam Clin Pharmacol, 19: 465-472, 2005

[2] Upur H, Yusup A, Baudrimont I, Umar A, Berke B, and et al., "Inhibition of cell growth and cellular protein, DNA and RNA synthesis in human hepatoma (HepG2) cells by ethanol extract of Abnormal Savda Munziq of traditional Uighur medicine," Evid Based Complement Alternat Med, 2011: 251424, 2011.

[3] Yusup A, Upur H, Umar A, Berke B, Yimit D, and et al., "Abnormal Savda Munziq, an herbal preparation of traditional Uighur medicine, may prevent 1,2-dimethylhydrazine-induced rat colon carcinogenesis," Evid Based Complement Alternat Med, 2011: 152015, 2011.

[4] Amat N, Upur H, Ablimit A, Matsidik A, Yusup A and Kijjoa A, "Immunomodulatory effects of Abnormal Savda Munsiq, a traditional Uighur medicine, on the combined stress mice," J Ethnopharmacol, 122: 42-47, 2009.

[5] Stolze K, Ablimit A and Strasser A, "Effects on cell proliferation and EPR spin trapping studies of Abnormal Savda Munziq and its chromatographic subfractions," BMC Pharmacol, 10 (Suppl 1): A14, 2010.

[6] Yusup A, Upur H, Tursun X, Berke B, Baudrimont I and Moore N, "Study on mechanism of abnormal savda munziq flavonoids in induction of apoptosis of Hep2 cells," Zhongguo Zhong Yao Za Zhi, 32:1068-1071,2007 (In Chinese).

[7] Kim DY, Jung KH, Kim TH, Kim DW, Chang HJ, and et al, "Comparison of 5-fluorouracil/leucovorin and capecitabine in preoperative chemoradiotherapy for locally advanced rectal cancer," Int J Rad Oncol Biol Phys, 67: 378-384, 2007.

[8] Lee HS, Choi Y, Hur WJ, Kim HJ, Kwon HC, and et al., "Pilot study of postoperative adjuvant chemoradiation for advanced gastric cancer: adjuvant 5-FU/cisplatin and chemoradiation with capecitabine,” World J Gastroenterol, 12: 603-607, 2006.

[9] Aikemu A, Umar A, Yusup A, Upur H, Berké B, and et al., "Immunomodulatory and antitumour effects of abnormal Savda Munziq on S180 tumour-bearing mice," BMC Complement Altern Med, 12: 157, 2012.

[10] Upur H, Yusup A, Baudrimont I, Umar A, Berke B, and et al., "Inhibition of cell growth and cellular protein, DNA and RNA synthesis in human hepatoma (HepG2) cells by ethanol extract of Abnormal Savda Munziq of traditional Uighur medicine," Evid Based Complement Alternat Med, 2011: 251424, 2011.

[11] LU R,Conrad P, and Yacine H, "Antitumor activity of mushroom polysaccharides:a reviw," Food Funct, 3(11):1118-1130, 2012.

[12] Longley DB, Harkin DP, and Johnston PG: 5-fluorouracil mechanisms of action and clinical strategies," Nat Rev Cancer, 3: 330-338,2003

[13] Finkel T and Holbrook NJ, "Oxidants, oxidative stress and the biology of ageing," Nature, 408: 239-247, 2000.

[14] Harman D, "Aging: a theory based on free radical and radiation chemistry," J Gerontol, 11: 298-300, 1956.

[15] Inal ME, Kanbak G and Sunal E, "Antioxidant enzyme activities and malondialdehyde levels related to aging," Clin Chim Acta 305: 75-80, 2001.

[16] Andersen HR, Nielsen JB, Nielsen F and Grandjean P. "Antioxidative enzyme activities in human erythrocytes," Clin Chem, 43: 562-568, 1997.

[17] Arai K, Maguchi S, Fujii S, and et al., "Glycation and inactivation of human $\mathrm{Cu}-\mathrm{Zn}$ - superoxide dismutase. Identification of the in vitro glycated sites," J Biol Chem, 262: 16969-16972, 1987.

[18] Gupta V, Lahiri SS, Sultana S, Tulsawani RK and Kumar R, "Anti-oxidative effect of Rhodiola imbricata root extract in rats during cold, hypoxia and restraint (C-H-R) exposure and post-stress recovery," Food Chem Toxicol, 48: 1019-1025, 2010.

[19] Ku SK, Seo BI, Park JH, Park GY, Seo YB, and et al., "Effect of Lonicerae Flos extracts on reflux esophagitis with antioxidant activity," World J Gastroenterol, 15: 4799-4805, 2009.

[20] Le Leu RK, Hu Y, Brown IL, Woodman RJ and Young GP, "Synbiotic intervention of Bifidobacterium lactis and resistant 
starch protects against colorectal cancer development in rats," Carcinogenesis, 31: 246-251, 2010

[21] Zhu LP, Yin Y, Xing J, Li C, Kou L, and et al., "Therapeutic efficacy of Bifidobacterium longum-mediated human granulocyte colony-stimulating factor and/or endostatin combined with cyclophosphamide in mouse-transplanted tumors," Cancer Sci, 100: 1986-1990, 2009. 